\title{
Spectroscoplc evidence against nitric acid trihydrate In polar stratospheric clouds
}

\author{
Owen B. Toon* a Margaret A. Tolbert $\dagger$
}

* NASA Ames Research Center, Moffett Field, California 94035, USA + Department of Chemistry and Blochemistry and CIRES.

University of Colorado, Boulder, Colorado 80309, USA

HeTerogeneous reactions on polar stratospheric clouds (PSCs) play a key role in the photochemical mechanism thought to be responsible for ozone depletion in the Antarctic and the Arctic ${ }^{1,2}$. Reactions on PSC particles activate chlorine to forms that are capable of photochemical ozone destruction, and sequester nitrogen oxides $\left(\mathrm{NO}_{x}\right)$ that would otherwise deactivate the chlorine $\mathrm{e}^{3,4}$. Although the heterogeneous chemistry is now well established, the composition of the clouds themselves is uncertain. It is commonly thought that they are composed of nitric acid trihydrate $^{3}$, although observations have left this question unresolved $^{3-14}$. Here we reanalyse infrared spectra of type I PSCs obtained in Antarctica in September 1987 15.16 , using recently measured optical constants of the various compounds that might be present in PSCs ${ }^{17}$. We find that these PSCs were not composed of nitric acid trihydrate but instead had a more complex composition, perhaps that of a ternary solution. Because cloud formation is sensitive to their composition, this finding will alter our under- 


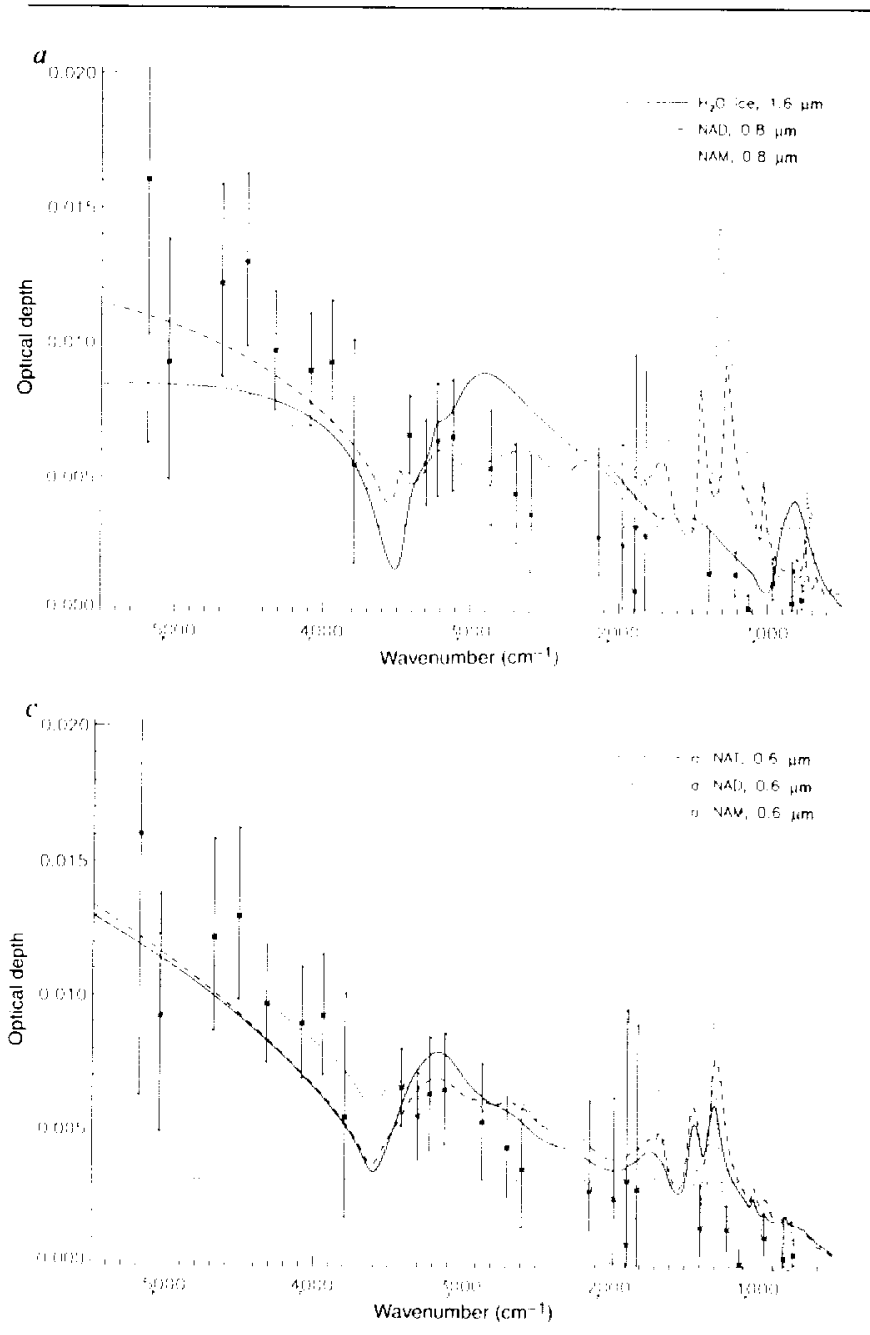

FIG. 1 a, Computed spectra for ice, NAM and NAD are compared with an observed spectrum (data points, with error bars) of PSCs. The numbers in the key correspond to the radius of the log-normal distribution used in the calculated spectrum. The radius shown is that which gave the best fit to the observations for that composition. The dotted horizontal line is an estimate of the instrument noise level ${ }^{15}$. Data below that level may represent random errors in the measurements, rather than
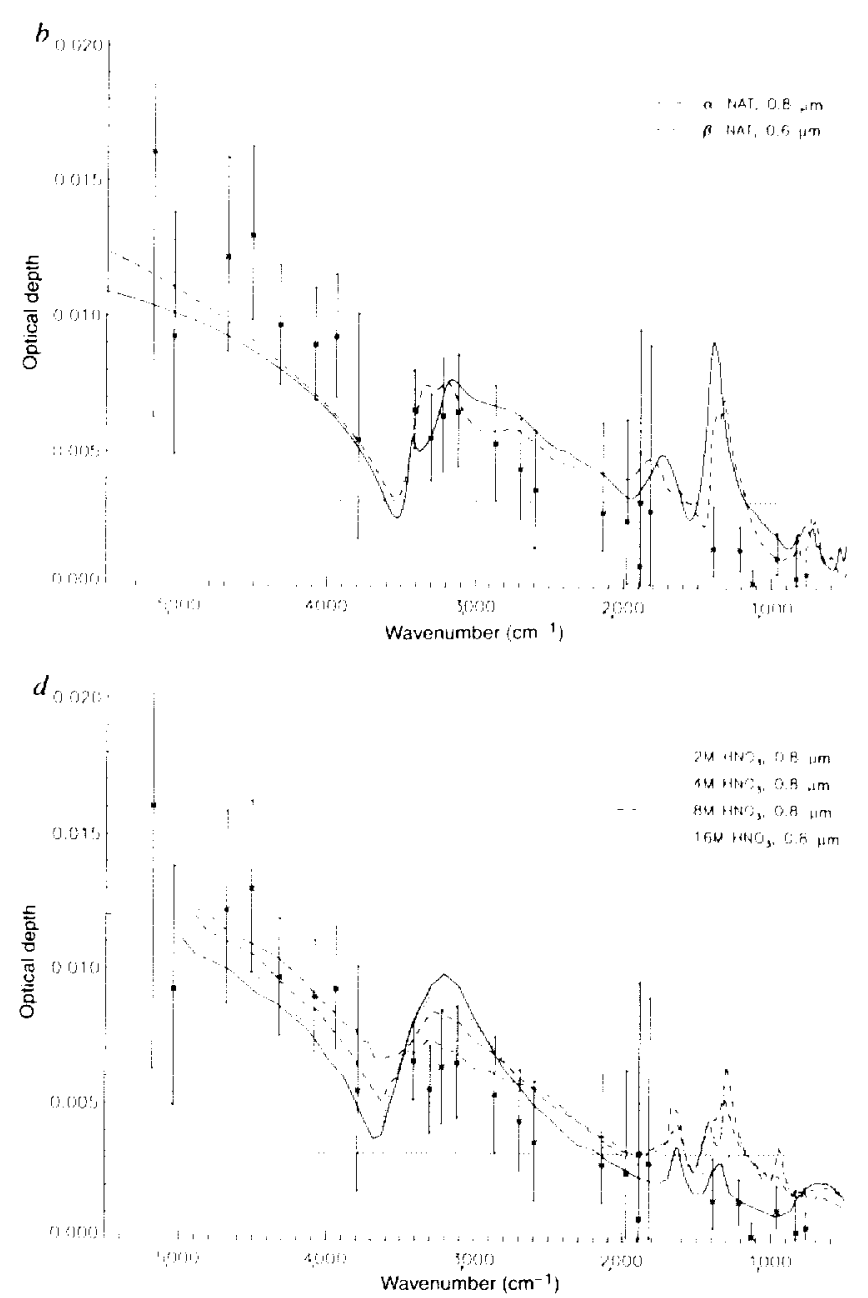

a true value of the infrared optical depth. The error bars are $1 \sigma$ based on all of the frequencies included within a spectral window. $b$. As for $a$ except that $\alpha$-NAT and $\beta$-NAT are considered. $c$, As for a except that aNAT, a-NAD and a-NAM are considered. $d$, As for a except that computed spectra using room-temperature optical constants ${ }^{26}$ for $2 \mathrm{M}(12 \mathrm{wt} \%)$ $4 \mathrm{M}(22 \mathrm{wt} \%), 8 \mathrm{M}(40 \mathrm{wt} \%)$ and $15.7 \mathrm{M}(70 \mathrm{wt} \%)$ liquid nitric acid solutions are compared with the observed spectrum.

from gaseous absorption, using a Michelson interferometer. Within each spectral window 7-500 frequencies were sampled. The typical standard deviation of clear spectra in the windows was used to establish an instrument noise level ${ }^{15}$. By comparing spectra from clear and cloudy regions, a mean cloud optical depth was determined in each spectral window. Calculations demonstrated that these PSCs could not be made of water ice ${ }^{15}$. However, lack of available optical constants at that time prevented a determination of whether the particles were made of crystalline forms of nitric acid, which are generally the favoured candidates for PSC composition ${ }^{3.5 .7 .9 .22}$. Here we reanalyse these spectra using newly measured optical constants of water ice, nitric acid trihydrate (in the $\alpha$-NAT and $\beta$-NAT forms), nitric acid dihydrate (NAD), and nitric acid monohydrate (NAM) ${ }^{17}$. We also investigate amorphous solid solutions, a-NAT, a-NAD and a-NAM, with compositions that crystallize upon heating to form hydrates.

Because the various observed spectra are similar, we reanalyse an average of four spectra for 21 September 1987 (Fig. 1). Two spectra were obtained at 14:08 14:10 universal time (UT) near $105 \mathrm{~W}$ longitude and $84 \mathrm{~S}$ latitude, and two spectra were obtained at $14: 33 \quad 14: 36$ UT at $90^{\circ} \mathrm{W}$ and $87 \mathrm{~S}$. During the optical-depth analysis. these spectra were normalized to clear- 
sky spectra taken at nearby locations ${ }^{15}$. We chose these four spectra because their signal-to-noise ratio was high relative to other spectra. The high signals were due to the relatively large cloud optical depths, and because the Sun was close to the horizon during the measurements which increased the optical path length through the cloud. Lidar data suggest these PSCs were at $15.18 \mathrm{~km}$ altitude ${ }^{15}$. Analyses of the observed spectral dependence of the infrared optical depths suggests that the radius of these particles was $\sim 0.8 \mu \mathrm{m}$, their number concentration was $\sim 1 \mathrm{~cm}^{-3}$, and their mass mixing ratio was $\sim 10$ p.p.b. (ref. 15). None of these derived particle properties is very sensitive to particle composition, as they are based on a portion of the spectrum in which scattering dominates, as discussed further below. Observations of the mass of sulphuric acid particles above Antarctica in 1987 suggest that $\sim 5 \%$ of the mass of the PSCs could be sulphuric acid ${ }^{23}$. There were $\sim 8$ sulphuric acid particles per $\mathrm{cm}^{3}$ in the altitude range of the PSCs observed ${ }^{24}$. The smaller number of PSC particles observed may represent lack of sensitivity of the infrared observations to small particles, rather than incomplete nucleation of the sulphuric acid particles to form PSC particles. As the sulphuric acid particles have low mass they do not have an observable effect on the infrared spectra, and because they should be uniformly distributed their spectral features would be removed when our PSC spectra are normalized to clear-sky spectra to obtain the PSC optical depths ${ }^{15}$.

The observed spectrum at frequencies above $3.500 \mathrm{~cm}^{-1}$ (Fig. 1) is dominated by scattering of the solar beam. because micrometre-sized ice and nitric acid particles are nearly transparent at these frequencies. The slope of the spectrum at these frequencies is controlled by the particle size. Below $3,500 \mathrm{~cm}^{-1}$ the spectrum is sensitive to particle size, but also to the optical constants of the materials because ice and nitric acid particles are absorbing at these frequencies. Varying the number of cloud particles results in a wavelength-independent scaling factor for the optical depths. We performed Mie scattering calculations of the optical depth for each set of optical constants, assuming a log-normal size distribution for the particles with a standard deviation $\sigma=1.5$ and variable mode size. The optical depths for a given mode size were then scaled to the data, and the mode size varied to obtain the least mean-square deviation between the calculated and observed optical depths.

Figure la shows that the observed PSCs are not composed of ice. Ice has strong absorption features near 3,000 and $800 \mathrm{~cm}^{-1}$. which are absent in the data. We have minimized the strength of these features in the calculated optical depth spectra by making the ice particles relatively large. Increasing the particle size to produce a still better fit at $3,000 \mathrm{~cm}^{-1}$ produces a poorer $\mathrm{fit}^{15}$ at frequencies above $4,000 \mathrm{~cm}^{-1}$. NAM has absorption features between 3,000 and $2,000 \mathrm{~cm}^{-1}$, and near $1,200 \mathrm{~cm}^{-1}$ that do not appear in the data, and NAM is not absorbing enough near $3,300 \mathrm{~cm}^{-1}$ to fit the data (Fig. $1 a$ ). NAD does not fit the spectrum well between 3,500 and $2,000 \mathrm{~cm}^{-1}$, and has bands near $1,400 \mathrm{~cm}^{-1}$ and $1,200 \mathrm{~cm}^{-1}$ that are not observed (Fig. $1 a$ ). Figure $1 b$ shows that $\alpha$-NAT and $\beta$-NAT have strong bands at 1,200 and $1,400 \mathrm{~cm}$ ' which are not observed. Figure $1 c$ shows that a-NAT and a-NAD also have strong bands near 1,400 and $1,200 \mathrm{~cm}^{-1}$ that are not observed. In general, all of the constituents whose spectra have been calculated have strong absorption bands that are not observed. In particular, NAT has bands that are calculated to lie more than two standard deviations above the observed spectra.

We performed many additional comparisons between observed and calculated spectra. We varied the particle size and the width of the size distribution, tried mixtures in which different composition particles either coexisted or were in cloud layers at different altitudes, explored shell and core combinations of materials, and computed spectra for particles with oblate or prolate shapes. None of these attempts resulted in a better match between calculated and observed spectra than those shown in Fig. 1.
The calculated spectrum of a-NAM with particle sizes of $0.6 \pm 0.2 \mu \mathrm{m}$ does agree with the observations (Fig. 1c) because a-NAM has relatively low imaginary refractive indices between its major absorption bands from 1,000 to $1,500 \mathrm{~cm}^{-1}$. The major objection to a-NAM as a constituent of PSCs is that its nitric acid vapour pressure is too large for it to occur in the stratosphere $^{10}$. Moreover, we have examined the observed infrared extinction in the region near $1,300 \mathrm{~cm}^{-1}$, and do not find evidence for the strong band which a-NAM has at that frequency.

Recent laboratory and theoretical work points to the possible existence of ternary solutions of nitric acid, sulphuric acid and water in PSCs ${ }^{12} 14$. Given the low temperature at the locale of our observations (near or below $192 \mathrm{~K}$ ), as well as the dehydrated and denitrified conditions of the lower stratosphere in September 1987, theory suggests that supercooled ternary solutions would be $\sim 5 \mathrm{wt}^{1} \%$ sulphuric acid and $30.50 \mathrm{wt} / \%$ nitric acid ${ }^{14}$. The refractive indices for amorphous nitric acid compounds discussed in this Letter, which should be similar to those of supercooled nitric acid ${ }^{25}$, are available only for solutions more concentrated than $50 \mathrm{wt} / \%$. But room-temperature refractive indices for less-concentrated nitric acid solutions are available ${ }^{26}$, and Fig. 1d shows that these provide a much better fit to the observations than any of the materials investigated in Fig. Ia $c$ As the nitric acid concentration declines, as shown in Fig. $1 d$. the nitrate bands near $1,2001.400 \mathrm{~cm}{ }^{1}$ decrease in strength. A solution less concentrated than $4 \mathrm{M}(22 \mathrm{wt} \%)$ is required to fit the spectrum. However, the $3,000 \mathrm{~cm}^{-1}$ water band increases in strength as the nitric acid concentration declines. A solution more concentrated than $4 \mathrm{M}$ is required to fit the spectrum above $3,000 \mathrm{~cm}^{-1}$. At present no optical constants are available either for ternary solutions, or for binary nitric acid solutions with relevant compositions at stratospheric temperatures. Studies of sulphuric acid binary solutions (which may have similarities to nitric acid solutions), have found that the infrared spectra of solutions containing $<50 \%$ sulphuric acid are strongly temperature dependent $t^{27}$. Quantitative determination of the nitric acid concentration from the observed atmospheric spectra will require measurements of the low-temperature optical constants of ternary mixtures. Additional atmospheric spectra would also be useful to search for changes in the composition of the particles caused by variations in environmental conditions ${ }^{14}$.

It has been suggested that there is a hemispheric asymmetry in PSC composition, with NAT clouds existing in the Antarctic. but other compounds in the Arctic ${ }^{5}$. However, our results show that the type I PSCs over Antarctica during September 1987, a period with significant ozone loss, were not composed of NAT. Our results imply that it is much more difficult to form NAT than thought previously. Of course, we cannot rule out NAT formation at other times and places than the ones we studied.

The extent of future ozone loss is controlled in part by the ability of PSCs to permanently remove $\mathrm{NO}_{x}$ from the stratosphere, so-called 'denitrification'. Large particles with rapid sedimentation rates form when a small fraction of the pre-existing particles nucleate to form crystals such as NAT. In contrast, small particles form when liquid PSCs grow within all of the stratospheric aerosols ${ }^{14}$. Most theories for future ozone loss assume that NAT is the principal component of PSCs. If so, stratospheric cooling during the next century due to the greenhouse effect may trigger an increase in the formation of NAT clouds in the colder Arctic stratosphere. The resulting enhanced sedimentation of nitric acid has been predicted to reduce the sensitivity of stratospheric ozone to anthropogenic perturbations in nitrogen oxides ${ }^{2 *}$. But if NAT is as difficult to form as our study jmplies, then only smaller ternary solution particles may form as the stratosphere cools. These smaller particles would not efficiently remove nitric acid from the stratosphere at temperatures above the frost point.

Massive chlorine-catalysed ozone loss such as observed in the Antarctic ozone hole may require denitrification. Denitrification 
of the stratosphere by ice clouds (type II PSCs) is inefficient if type I PSCs contain NAT ${ }^{29}$. However, the higher vapour pressures of ternary solutions would greatly speed the transfer of nitric acid from type I to type II PSCs. Therefore the lack of evidence for type I PSCs containing NAT implies that nitrogen removal from the stratosphere may be more closely related to the formation of ice particles than it is to nitric acid particles ${ }^{3}$.

It has also been suggested that enhancements in the concentrations of stratospheric nitric acid and water vapour from future fleets of supersonic aircraft might lead to significant expansions of type I PSCs composed of NAT ${ }^{30}$. But the variation in PSC abundance for a given change in nitric acid vapour supply may be quite different for NAT than for other PSC components. NAT formation should occur at a definite onset temperature, whereas particles composed of ternary solutions increase gradually in size and coverage as the concentration of nitric acid vapour increases. Assuming a NAT composition, regions currently near the NAT formation point would experience a sudden drastic increase in PSC abundance if the nitric acid vapour concentration was increased past the threshold value. In contrast. only a modest change in the abundance of ternary aerosols would occur as the existing aerosols would swell as conditions changed. In areas far from the NAT threshold, small changes in the nitric acid vapour abundance would have no effect on NAT aerosols, but the ternary aerosols would change their size and hence their potential to cause ozone loss.

Owing to the complexity of stratospheric chemistry and PSC microphysics, it is not possible to anticipate the quantitative changes that might occur in theoretical studies which have assumed PSCs are composed of NAT. But it is clear that the properties of other possible PSC constituents are sufficiently different from those of NAT that future theories should not treat PSCs as though they were composed only of NAT. It is also clear that we need to understand better the environmental condjtions that control PSC composition.

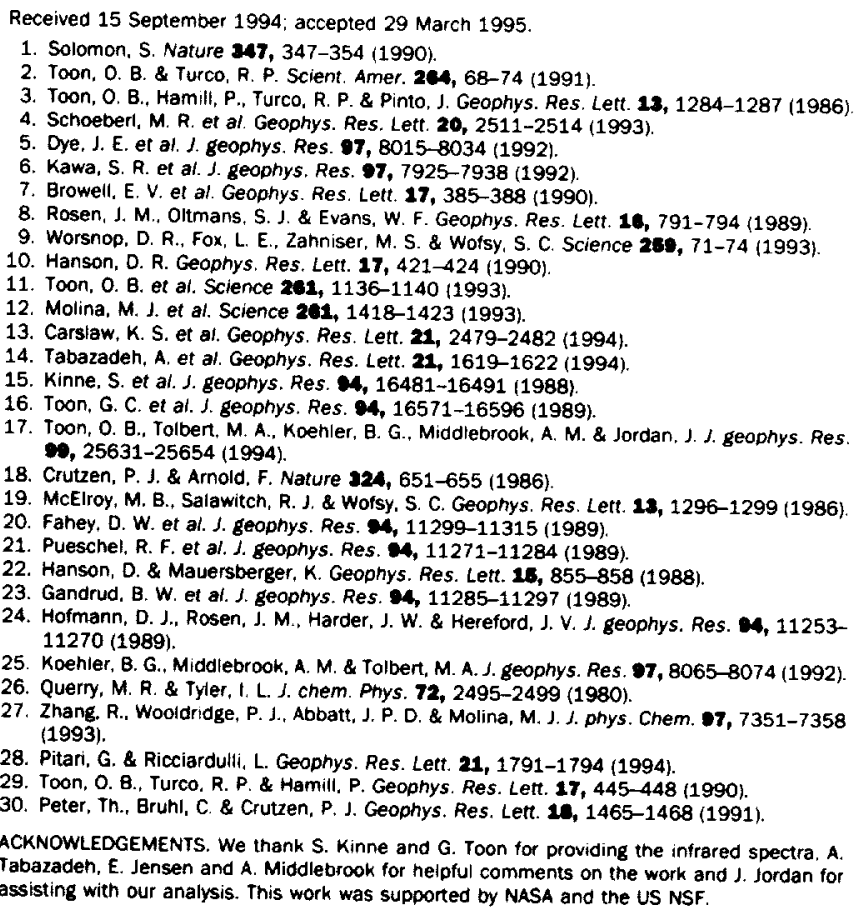

